\title{
CORRIGENDUM
}

\section{Non-Western immigrant children have lower 25-hydroxyvitamin D than children from Western families - CORRIGENDUM}

\author{
Jessica A Omand, Pauline B Darling, Patricia C Parkin, Catherine S Birken, \\ Marina Khovratovich, Kevin E Thorpe, Sarah Carsley, Julie DeGroot and \\ Jonathon L Maguire on behalf of the TARGet Kids! Collaboration
}

First published online 28 June 2013

doi:10.1017/S1368980013001328 published online by Cambridge University Press 24 May 2013

Affiliations for Jessica Omand and Pauline B Darling should both include the Keenan Research Centre, Li Ka Shing Knowledge Institute, St. Michael's Hospital, Toronto, ON, Canada.

Affiliations should read:

Jessica A Omand ${ }^{1,2,7}$

${ }^{1}$ Department of Nutritional Sciences, University of Toronto, Toronto, ON, Canada:

${ }^{2}$ Department of Pediatrics, St. Michael's Hospital, 61 Queen Street East 2nd Floor, Toronto, ON M5C 2T2, Canada:

${ }^{7}$ Keenan Research Centre, Li Ka Shing Knowledge Institute, St. Michael's Hospital, Toronto, ON, Canada:

Pauline B Darling ${ }^{1,7}$

${ }^{1}$ Department of Nutritional Sciences, University of Toronto, Toronto, ON, Canada:

${ }^{7}$ Keenan Research Centre, Li Ka Shing Knowledge Institute, St. Michael's Hospital, Toronto, ON, Canada:

\section{Reference}

Omand JA, Darling PB, Parkin PC, Birken CS, Khovratovich M, Thorpe KE, Carsley S, DeGroot J \& Maguire JL (2013) Non-Western immigrant children have lower 25-hydroxyvitamin D than children from Western families. Public Health Nutrition, published online 24 May 2013, doi:10.1017/\$1368980013001328. 